\title{
Generalized Optimal Velocity Model for Traffic Flow
}

\author{
Shiro Sawada円 \\ Department of Telecommunications, \\ Takuma National College of Technology, \\ Mitoyo, Kagawa 769-1192, Japan
}

\begin{abstract}
A generalized optimal velocity model is analyzed, where the optimal velocity function depends not only on the headway of each car but also the headway of the immediately preceding one. The stability condition of the model is derived by considering a small perturbation around the homogeneous flow solution. The effect of the generalized optimal velocity function is also confirmed with numerical simulation, by examining the hysteresis loop in the headway-velocity phase space, and the relation between flow and density of cars. In the model with a specific parameter choice, it is found that an intermediate state appears for the movement of cars, where the car keeps a certain velocity though the headway is short or long. This phenomenon is different from the ordinary stop-and-go state.
\end{abstract}

\footnotetext{
${ }^{1}$ e-mail: sawada@dt.takuma-ct.ac.jp
} 


\section{Introduction}

Traffic flow problem has been extensively studied from physical point of view. Fluid-dynamical model[1], cellular automaton model [2], and the carfollowing model [3] have been proposed and analyzed in detail to understand the mechanism of the traffic congestion on a freeway.

Toward a realistic model which explains the traffic flow dynamics, the optimal velocity (OV) model proposed by Bando, Hasebe, Nakayama, Shibata, and Sugiyama [|⿴囗十, , 5] has attracted considerable interest. Based on the secondorder differential equations, the model reveals the density pattern formation of the congested flow of traffic without introducing a time lag caused by the driver's response.

Although the OV model is shown to have a universal structure in spatiotemporal patterns in the congestion, most of the analyses of the model have been done in the case where the optimal velocity function depends only on the headway of each car. One of the approaches to generalize the OV model is that the backward reference function is introduced [6]. Another approach to extend the OV model is to take into account the next-nearest-neighbor interaction [7], where the optimal velocity function depends not only on the headway of each car but also on the headway of the immediately preceding one. The generalized optimal velocity function is determined by taking into account the driver's skill, experience, and psychological effect, so that it is expected to describe more realistic traffic flow.

The purpose of this paper is to analyze the generalized optimal velocity model proposed by Nagatani[0, 8]. This paper is organized as follows. In Section 2 the generalized optimal velocity model is reviewed and its stability condition around the homogeneous flow solution is derived without longwavelength approximation. In Section 3 numerical simulations are carried

out, in particular, the hysteresis loop in the phase space and the flow-density relation are examined. In Section 4 we reconsider our model to compare the result with the one in the original model. In the model with a specific parameter choice, it is found that an intermediate state appears for the movement of cars, which is different from the ordinary stop-and-go state.

\section{Generalized Optimal Velocity Model}

We first consider a dynamical model of the traffic flow given by

$$
\ddot{x}_{n}(t)=a\left(V\left(\Delta x_{n}(t), \Delta x_{n+1}(t)\right)-\dot{x}_{n}(t)\right),
$$


where $x_{n}(t)$ is the position of the $n$-th car at time $t, \Delta x_{n}(t)=x_{n+1}(t)-x_{n}(t)$ represents the headway of the car, and $a$ is the sensitivity. Thus $\Delta x_{n+1}(t)$ is the headway of the immediately preceding car. Here $n=1,2, \cdots, N$ is each car number with $N$ being the total number of vehicles. The driver's sensitivity $a$ is assumed to be independent of $n$. Throughout this paper, we will consider the periodic boundary condition with respect to the coordinate $x_{n}$ with period $L$.

At first, let us look for an appropriate form of the optimal velocity function to be suitable for our purpose. The driver sometimes pays attention to not only the headway but also the headway of the immediately preceding one. If the headway of the preceding car is short, the driver assumes that the forward driver decelerates, thus the driver decrease the optimal velocity even though the headway of his car is long enough. On the other hand, if the headway of the preceding car is long, the driver assumes that the forward driver accelerates, thus the driver increase the optimal velocity even though the headway of his car is short.

Let us look at Figure 1, which describes the original optimal velocity function in $\Delta x_{n}, \Delta x_{n+1}$, and $V$ space, where the numerical values of axes are not important here. The appropriate form of the function which satisfies the above requirement will be seen in Figure 2.
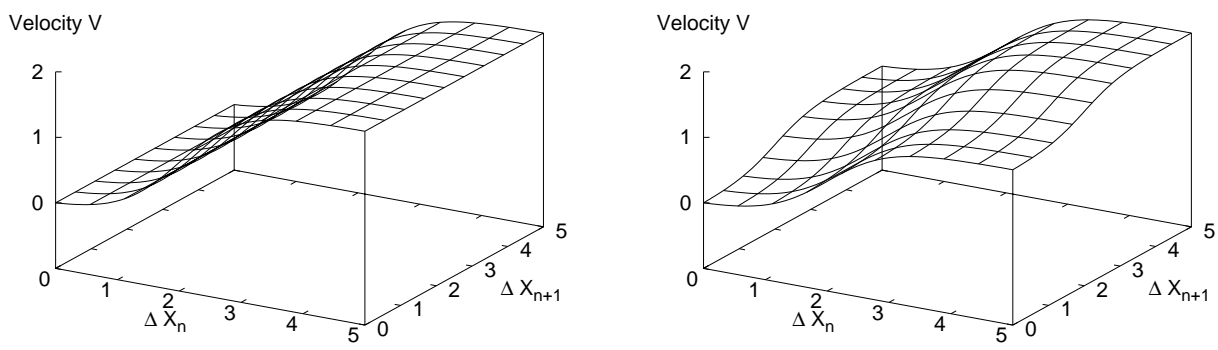

Figure 1: The original optimal ve- Figure 2: The generalized optimal locity function $V\left(\Delta x_{n}\right)$. velocity function $V\left(\Delta x_{n}, \Delta x_{n+1}\right)$.

As is already introduced in Ref. [7, 8], we can adopt the generalized optimal velocity function of the form

$$
V\left(\Delta x_{n}, \Delta x_{n+1}\right)=(1-p) V\left(\Delta x_{n}\right)+p V\left(\Delta x_{n+1}\right),
$$

where $p$ is assumed to be independent of $n$ and satisfies $0 \leqq p<\frac{1}{2}$ because the dominant part of the optimal velocity function should be $\Delta x_{n}$ dependent 
term. The advantage of the above form is to be able to find the effect of the additional term with varying $p$. In spite of introducing $p$ and $\Delta x_{n+1}$ dependent term, the above model provides exactly the same homogeneous flow solution as the original one without $p$ dependence. When $p=0$, the model reduces to the original one. According to the original OV function proposed by Bando et al. [4], we will take hyperbolic tangent function of the form

$$
V\left(\Delta x_{n}\right)=\tanh \left(\Delta x_{n}-2\right)+\tanh (2) .
$$

As we can see from eq.(2) with eq.(3), the generalized optimal velocity function has the required properties. In general, $p$ might depend on $n$ and also depend on time $t$. The numerical simulation can be still performed under these circumstances.

More generally, we can consider the form

$$
V\left(\Delta x_{n}, \Delta x_{n+1}\right)=U\left(\Delta x_{n}\right)+W\left(\Delta x_{n+1}\right),
$$

where $U$ and $W$ have the following properties: (i) monotonically increasing functions, (ii) they have upper bounds, and (iii) they satisfy $|U|>|W|$ as a realistic model.

Now, let us analyze the generalized OV model given by eq.(11) with eq.(任)

$$
\ddot{x}_{n}(t)=a\left(U\left(\Delta x_{n}(t)\right)+W\left(\Delta x_{n+1}(t)\right)-\dot{x}_{n}(t)\right) .
$$

As is easily seen from eq.(5), they have a homogeneous flow solution

$$
x_{n}^{(0)}(t)=b n+c t
$$

where $b=L / N$ and $c=U(b)+W(b)$. We examine the stability against a small perturbation $y_{n}(t)$ around the homogeneous flow solution (6). Substituting

$$
x_{n}(t)=x_{n}^{(0)}(t)+y_{n}(t)
$$

into eq.(5), the linearized equation to $y_{n}(t)$ is obtained as

$$
\ddot{y}_{n}(t)=a\left(g \Delta y_{n}(t)+h \Delta y_{n+1}(t)-\dot{y}_{n}(t)\right),
$$

where $\Delta y_{n}(t)=y_{n+1}(t)-y_{n}(t)$, and $g$ and $h$ is the derivative of $U$ and $W$ at $b$, respectively. The solutions to eq.(8) is given by the Fourier series as

$$
y_{n, k}(t)=\exp \left(i \alpha_{k} n+z t\right)
$$

where $\alpha_{k}=2 \pi k / N$ with $k=1,2, \cdots, N$ and $z$ satisfies

$$
z^{2}+a z-a\left(g\left(e^{i \alpha_{k}}-1\right)+h\left(e^{2 i \alpha_{k}}-e^{i \alpha_{k}}\right)\right)=0 .
$$


The stability condition is to find the condition $\operatorname{Re} z<0$ for all modes $\alpha_{k}$. After a short calculation, it is equivalent to find the relation

$$
F(Y) \equiv 32 h^{2} Y^{3}+16 h(g-3 h) Y^{2}+2\left((g-3 h)^{2}-2 a h\right) Y-a(g-h)<0
$$

holds for all modes $\alpha_{k}$, where we put $Y=\cos ^{2} \frac{\alpha_{k}}{2}$.

In order to proceed the analysis of stability condition, we will take the optimal velocity function adopted in eq.(2). Eq.(11) is rewritten by

$F(Y)=32 p^{2} f^{2} Y^{3}+16 p(1-4 p) f^{2} Y^{2}+2\left((1-4 p)^{2} f^{2}-2 a p f\right) Y-a(1-2 p) f<0$,

where $f=V^{\prime}(b)$. We can finally find that if the condition

$$
f<\frac{a}{2}(1+2 p) \text { and } p \leqq \frac{1}{2}
$$

is satisfied, eq.(12) holds for all modes $\alpha_{k}$. It should be emphasized that the stability condition (13) is derived without long-wavelength approximation. In the long-wavelength approximation, only the former condition in eq.(13) is derived from the stability analysis. The uniform solution is unstable if $f>\frac{a}{2}(1+2 p)$ or $p>\frac{1}{2}$. Comparing the result with the original OV model, we can conclude that the model is stabilized in the region $\frac{a}{2} \leqq f<\frac{a}{2}(1+2 p)$ by the effect of introducing the headway of the preceding car.

\section{$3 \quad$ Numerical Simulations}

To convince the analysis of stability condition for the generalized OV model, we will now solve

$$
\ddot{x}_{n}(t)=a\left((1-p) V\left(\Delta x_{n}\right)+p V\left(\Delta x_{n+1}\right)-\dot{x}_{n}(t)\right)
$$

numerically. In the simulation, $a=1$ is taken throughout this paper. As the density of cars $(\rho=N / L)$ varies and if $f>\frac{a}{2}(1+2 p)$ is satisfied, a homogeneous flow becomes unstable and makes a phase transition from free flow to congested one. Looking at the spatio-temporal pattern, the congested patters propagate backward. These characteristic features in the generalized model are the same as those appear in the original OV model.

One of the typical features of the model is that the movement of the car becomes the stop-and-go states in the congested region and the congested pattern is very stable. It is well understood by examining the hysteresis loop in the headway-velocity phase space. In numerical simulations, we take $N=100$ and $L=200$ as an example, where $f$ takes maximum value, because 
the congested region is of our interest and the result depends only on the density but not on the number of cars and the circuit length. The initial condition we considered here is the homogeneous flow with small fluctuation, i.e.

$$
x_{n}(0)=b n+y_{n}(0), \dot{x}_{n}(0)=c,
$$

where $y_{n}(0)$ is taken to be an uniform random distribution between -0.5 and 0.5 .

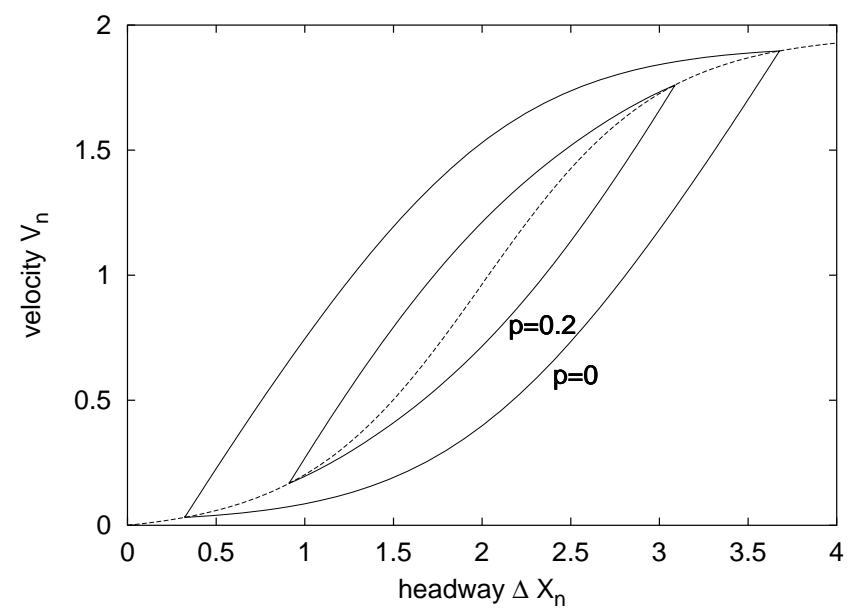

Figure 3: The orbit of a car in the headway-velocity phase space after the organization of the congestion for $p=0$ and $p=0.2$. The dashed curved line denotes the optimal velocity function.

Figure 3 shows the orbit of a particular car in the headway-velocity phase space with the parameter $p=0,0.2$ as an example. After about 1,000 time when the generation of the congestion is finished, the shape of the hysteresis loop obtained in Figure 3 never changes. Furthermore, the shape does not depend on the initial random distributions.

The numerical result shows that the effect of increasing $p$ seems to be equivalent to increasing the sensitivity $a$. Of course, this is also guessed from the analysis of the stability condition in eq.(13). However, as will be seen in Section 4, we will find that the change of the value of $p$ can not be compensated by rescaling the sensitivity $a$.

Examining the bottom end point $\left(\Delta x_{\mathrm{c}}, v_{\mathrm{c}}\right)=\left(\Delta x_{n}, \dot{x}_{n}\right)$ and the top end point $\left(\Delta x_{\mathrm{f}}, v_{\mathrm{f}}\right)=\left(\Delta x_{n}, \dot{x}_{n}\right)$ in the hysteresis loop, we can obtain the backward velocity of the congestion given by

$$
V_{\text {back }}=\frac{v_{\mathrm{f}} \Delta x_{\mathrm{c}}-v_{\mathrm{c}} \Delta x_{\mathrm{f}}}{\Delta x_{\mathrm{f}}-\Delta x_{\mathrm{c}}} .
$$


Numerical simulation shows that the backward velocity of the congestion increases as $p$ increases. We have also confirmed by simulating spatio-temporal patterns. The numerical results are listed in Table 1.

\begin{tabular}{|c|c|c|c|c|c|}
\hline$p$ & $\Delta x_{\mathrm{c}}$ & $v_{\mathrm{c}}$ & $\Delta x_{\mathrm{f}}$ & $v_{\mathrm{f}}$ & $V_{\text {back }}$ \\
\hline 0.0 & 0.32274 & 0.03152 & 3.67726 & 1.89653 & 0.14791 \\
\hline 0.1 & 0.62051 & 0.08319 & 3.37945 & 1.84485 & 0.31302 \\
\hline 0.2 & 0.91196 & 0.16787 & 3.08804 & 1.76019 & 0.49945 \\
\hline 0.3 & 1.18567 & 0.29206 & 2.81434 & 1.63600 & 0.68632 \\
\hline 0.4 & 1.46814 & 0.47750 & 2.53275 & 1.45136 & 0.86548 \\
\hline
\end{tabular}

Table 1: Numerical data for $p=0,0.1,0.2,0.3,0.4$.

Another important problem is to investigate the relation between the flux and the density, which is called fundamental diagram. The density $\rho$ of the cars is defined by $N / L$, where we choose $L=200$ and vary $N$ from 10 and 300 in the simulation. The flux $Q$ is defined by the number of cars passing by a position per unit time. The data was accumulated and averaged over during 20,000 time after first 1000 time. Numerical results are plotted in Figure 1 for $p=0,0.1,0.2,0.3$. Here we omitted to plot the data which overwrites the other data.

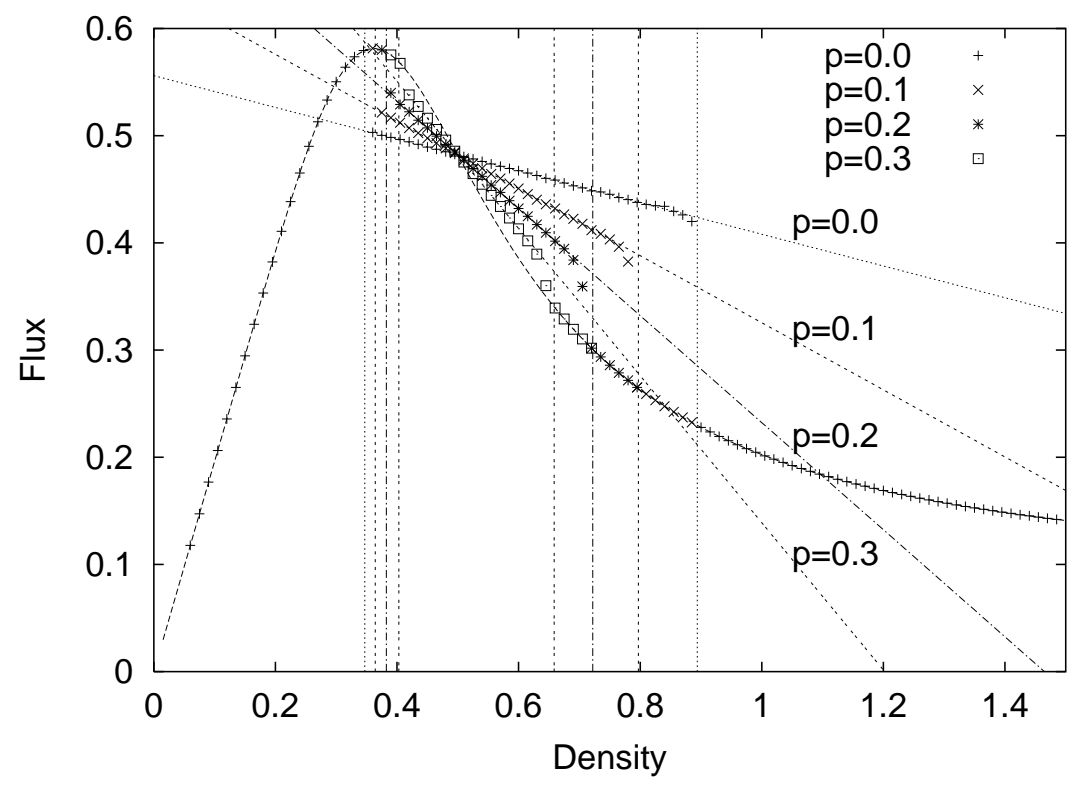

Figure 4: Flux-density fundamental diagram for $p=0,0.1,0.2,0.3$. 
In the homogeneous flow, the relation between flux $Q$ and density $\rho$ is given by

$$
Q=\rho V\left(\frac{1}{\rho}\right)=\rho\left(\tanh \left(\frac{1}{\rho}-2\right)+\tanh (2)\right) .
$$

In Figure 4 , it is represented by the dashed curved line. The numerical results agree with this line in the homogeneous flow region.

As is discussed in Ref. [5], the flux-density relation in the congested flow is evaluated from the data in Table 1. In the congested flow region, the relation between the density $\rho$ and the flux $Q$ is given by

$$
Q=\frac{v_{\mathrm{f}}-v_{\mathrm{c}}}{\Delta x_{\mathrm{f}}-\Delta x_{\mathrm{c}}}-V_{\mathrm{back}} \rho .
$$

Substituting the values in Table 1 into eq.(18), we obtain the $Q-\rho$ relation as in the Table 2. These lines are plotted in Figure 1 with the fundamental

\begin{tabular}{|c|c|}
\hline$p$ & $Q-\rho$ relation \\
\hline 0.0 & $Q=0.55597-0.14792 \rho$ \\
\hline 0.1 & $Q=0.63853-0.31302 \rho$ \\
\hline 0.2 & $Q=0.73174-0.49945 \rho$ \\
\hline 0.3 & $Q=0.82518-0.68632 \rho$ \\
\hline 0.4 & $Q=0.91475-0.86548 \rho$ \\
\hline
\end{tabular}

Table 2: Flux-density relation in congested flow for $p=0,0.1,0.2,0.3,0.4$.

diagram. In Figure 4 the vertical lines represent the boundaries for the stability condition given by eq.(13) in the case of $p=0,0.1,0.2,0.3$, where the same dashed line is used as the one used to draw the predicted line in eq.(18). The numerical results for various $p$ values are good agreement with the predicted lines in Table 2 .

We will summarize the effect of the $\Delta x_{n+1}$ dependent term in fundamental diagram. In the homogeneous flow region, there is no effect in the flux-density relation, because $p$ dependence in the generalized OV function disappears in the case of homogeneous flow, as is seen in eq.(2). In the congested region, the flow increases as $p$ increases if $\rho<\frac{1}{2}$ and inversely the flow decrease as $p$ increase if $\rho>\frac{1}{2}$. The reason is as follows. When the density is low, the average of the headway is long. If $\Delta x_{n+1}$ is long, the larger value of the velocity than that of the case without $\Delta x_{n+1}$ dependence is allowed. Hence the flow increases by taking into account the headway of the immediately preceding car. Inversely, when the density is high, i.e. $\rho>\frac{1}{2}$, the small value of the velocity is taken compared with the case of $p=0$, because $\Delta x_{n+1}$ is 
short. Hence the flow decreases by taking into account the headway of the immediately preceding car.

\section{Rescaled Model}

As far as the stability condition of traffic flow is concerned, our analytical result of eq.(13) means that the effect of the $\Delta x_{n+1}$ dependent term can be compensated by rescaling the sensitivity $a$. Thus we can rewrite the generalized OV model as

$$
\ddot{x}_{n}(t)=a\left(\frac{1-p}{1+2 p} V\left(\Delta x_{n}\right)+\frac{p}{1+2 p} V\left(\Delta x_{n+1}\right)-\frac{1}{1+2 p} \dot{x}_{n}(t)\right) .
$$

Now the stability condition of the above model is given by $f<\frac{a}{2}$ which is independent of $p$. By investigating the hysteresis loop in this model with various values of $p$, we can clarify the effect of the headway of the preceding car. Numerical simulation can be performed in the same way as in Section 3. Obtained numerical data for the headway and velocity of a particular car is plotted in Figure 5 .

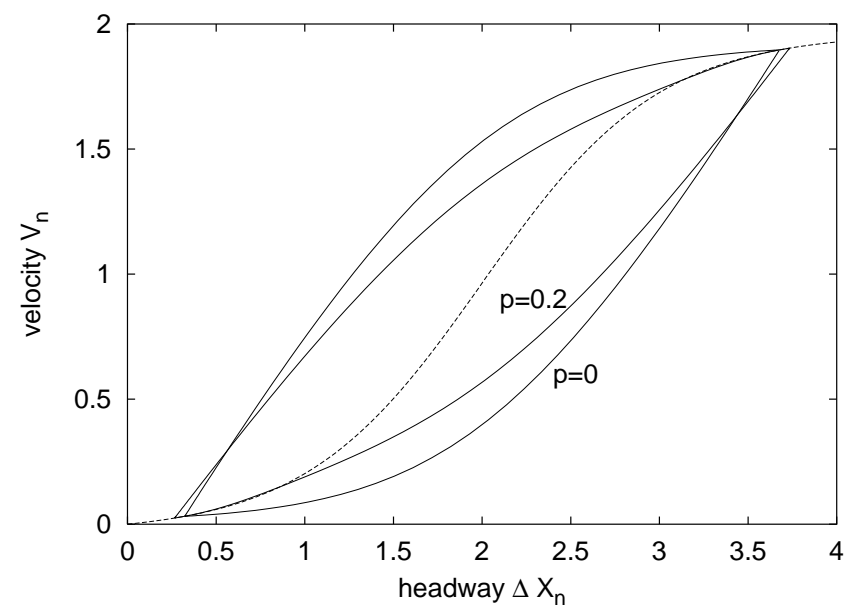

Figure 5: Hysteresis loops for $p=0$ and $p=0.2$.

We can find that the effect of the $\Delta x_{n+1}$ dependent term can not be compensated by rescaling the sensitivity $a$. The effect of $p$ is now easily understood. Compared with $p=0$, shorter value of the minimum of the headway is allowed, and also longer value of the maximum of the headway is allowed. Furthermore, when the car accelerates, larger value of the velocity 
can be taken even if the headway is short. Inversely, when the car decelerates, smaller optimal velocity is taken even if the headway is long enough.

The above characteristic feature in the phase space holds for various $p$ values as long as $p<\frac{1}{2}$. If we take $p>\frac{1}{2}$, the numerical simulation shows that the cars take over the cars ahead, thus it is not realistic. Of course, a room to change the form of OV function is left, but we will not consider it here.

It is found that the model give by eq.(19) has a different feature in the unstable state when we take $p=\frac{1}{2}$. Figure 6 shows the hysteresis loop for $p=\frac{1}{2}$ after some relaxation time, e.g. about $10^{8}$ time in the simulation. More precisely, two independent hysteresis loops appear for two successive cars. In Figure 6, the line and the dotted line are the hysteresis loops for the odd car number and even car number, respectively. This hysteresis loop is well understood when the orbit of the car is shown in the $\left(\Delta x_{n}, \Delta x_{n+1}, \dot{x}_{n}\right)$ phase space. Figure 7 shows the orbit of two successive cars in the phase
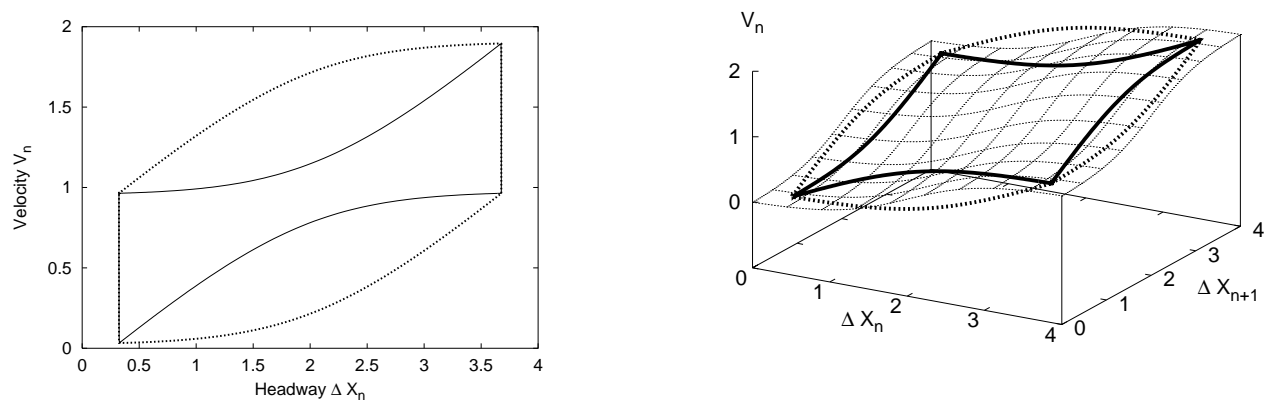

Figure 6: Hysteresis loops for $p=\frac{1}{2}$ Figure 7: Hysteresis loops for $p=\frac{1}{2}$ in $\left(\Delta x_{n}, \dot{x}_{n}\right)$ space. $\quad$ in $\left(\Delta x_{n}, \Delta x_{n+1}, \dot{x}_{n}\right)$ space.

space. The simulation shows that all of the vehicles sweep the same orbit as the one in Figures 6 and 77. We have examined the model with various initial configurations such as random distributions, different number of cars $N$ and circuit length $L$. The above result does not depend on the initial distributions and the parameters.

Figure 8 and 9 show the headways and the velocities for all vehicles, respectively, in the case of $N=100$ and $L=200$ at $10^{8}$ time, starting with arbitrary random distributions with zero velocities. Numerical simulations show that the characteristic feature does not depend on the initial distributions and the parameters. We can find that an intermediate state appears for the movement of cars, which is different from the ordinary stop-and-go 

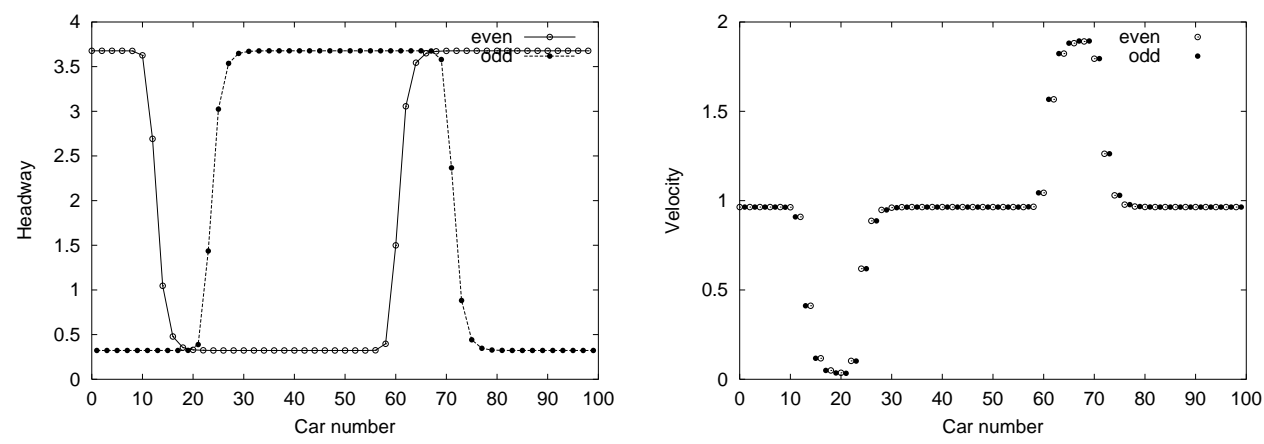

Figure 8: Headways for all vehicles Figure 9: Velocities for all vehicles at $10^{8}$ time with $N=100, L=200$. at $10^{8}$ time with $N=100, L=200$.

state. Numerically, the state corresponds to the points $\left(\Delta x_{n}, \Delta x_{n+1}, \dot{x}_{n}\right)=$ $(3.677,0.323,0.964)$ and $\left(\Delta x_{n}, \Delta x_{n+1}, \dot{x}_{n}\right)=(0.323,3.677,0.964)$ in the phase space as is given in Figure 7. Thus the intermediate state means that the car can keep a certain velocity (0.964) though the headway is short (0.323) or long (3.677) with holding $\Delta x_{n}+\Delta x_{n+1}=4$. The appearance of the intermediate state is universal in the sense that it does not depend on the initial distributions, the number of cars $N$ and the length $L$. It should be noted that this state never appears if $p<\frac{1}{2}$.

This characteristic feature is understood by considering the equation for the headway which is given by

$$
\Delta \ddot{x}_{n}=\frac{a}{1+2 p}\left((1-2 p) V\left(\Delta x_{n+1}\right)+p V\left(\Delta x_{n+2}\right)-(1-p) V\left(\Delta x_{n}\right)-\Delta \dot{x}_{n}\right) \text {. }
$$

If $p=\frac{1}{2}$ is chosen, the first term in the right hand side of eq.(20) vanishes and hence $\Delta x_{n+1}$ dependence disappears. One might expect that the intermediate state is understood by the nonlinear analysis near the critical point $a=a_{c}$. Following the analysis by Komatsu and Sasa[9], the equation for the headway is derived near the critical point and the modified Korteweg-de Vries(MKdV) equation and its higher-oder corrections is obtained by introducing a small scaling parameter $\epsilon=\sqrt{\left(a_{c}-a\right) / a_{c}}$. However, the equation near the critical point in the case of $p=\frac{1}{2}$ is exactly the same as the one in the case of $p=0$ up to the order $\epsilon$. Because the difference in eq.(20) with $p=0$ and $p=\frac{1}{2}$ near the critical point is that the Fourier mode with $p=\frac{1}{2}$ is just twice that of the other one with $p=0$. Since our analysis is performed far from the critical point, the higher-oder corrections or non-perturbative effect should be considered to understand the intermediate state. 


\section{$5 \quad$ Summary and Discussion}

We have analyzed the stability of the generalized optimal velocity model where the optimal velocity function depends not only on the headway of each car but also on the headway of the immediately preceding one. The effect of the newly introduced $\Delta x_{n+1}$ dependent term was examined by numerical simulation. In particular, the hysteresis loop in the phase space and the flux-density relation were examined in detail by taking the various values of the parameter.

We found that the effect of the $\Delta x_{n+1}$ dependent term can not be compensated by rescaling the sensitivity $a$. In the model with the specific parameter choice $p=\frac{1}{2}$, we found that the intermediate state appears for the movement of cars, which is different from the ordinary stop-and-go state. Numerical simulation shows that the appearance of the intermediate state is universal because it does not depend on the initial conditions.

We would expect that the model is related with the exact solution given by Jacobi's elliptic function[10]. It is interesting to examine the differencedifferential equation

$$
\dot{x}_{n}(t+\tau)=V\left(\Delta x_{n}(t), \Delta x_{n+1}(t)\right) .
$$

The details of the numerical simulation and the analysis of the underlining mathematical structure of the generalized optimal velocity model are under study.

\section{Acknowledgment}

The author would like to thank S. Tadaki for informing of the Refs. [7, 8]. The part of the numerical computation in this work was carried out at Yukawa Institute Computer Facility.

\section{References}

[1] M.J.Lighthill and G.B. Witham, Proc. R. Soc. London A 299 (1955) 317.

[2] S. Wolfram, Rev. Mod. Phys. 55 (1983) 601; S. Wolfram, Theory and Applications of Cellular Automata (World Scientific, Singapore, 1986); K. Nagel and M. Schreckenberg, J. Phys. I France 2 (1992) 2221; K. Nagel and H.J. Herrmann, Physica A 199 (1993) 254; S. Yukawa, M. Kikuchi and S. Tadaki, J. Phys. Soc. Japan 63 (1994) 3609; K. Nagel, Phys. Rev. E 53 (1996) 4655. 
[3] L.A. Pipes, J. Appl. Phys. 24 (1953) 274; G.F. Newell, J. Opns. Res. Soc. 9 (1961) 209; D.C. Gazis, R. Herman and R.W. Rothery, J. Opns. Res. Soc. 9 (1961) 545.

[4] M. Bando, K. Hasebe, A. Nakayama, A. Shibata, and Y. Sugiyama, Phys. Rev. E 51 (1995) 1035; M. Bando, K. Hasebe, A. Nakayama, A. Shibata, and Y. Sugiyama, Japan J. Indust. Appl. Math, 11 (1994) 203.

[5] M. Bando, K. Hasebe, A. Nakayama, A. Shibata, and Y. Sugiyama, J. Phys. I France 5 (1995) 1389.

[6] H. Hayakawa and K. Nakanishi, Phys. Rev. E 57 (1998) 3839.

[7] T. Nagatani, Phys. Rev. E 60 (1999) 6395.

[8] T. Nagatani and M. Muramatsu, Proceedings of the 7th Symposium on Simulation of Traffic Flow (in Japanese).

[9] T.S. Komatsu and S. Sasa, Phys. Rev. E 52 (1995) 5574.

[10] K. Hasebe, A. Nakayama and Y. Sugiyama, Phys. Lett. A 259 (1999) 135; Y. Igarashi, K. Itoh and K. Nakanishi, J. Phys. Soc. Japan, 68 (1999) 791; Y. Igarashi, K. Itoh, K. Nakanishi, K. Ogura, K. Yokokawa, patt-sol/9908002; K. Nakanishi, patt-sol/9909005. 